\title{
Post tuberculosis radiological sequelae in patients treated for pulmonary and pleural tuberculosis at a tertiary center in Pakistan
}

\author{
Syed Muhammad Zubair ${ }^{1}$, Mariyam Gohar Ali², Muhammad Irfan' \\ ${ }^{1}$ Section of Pulmonary and Critical Care Medicine, Department of Medicine, Aga Khan University Hospital, Karachi; \\ ${ }^{2}$ Department of Pulmonary and Critical Care Medicine, Dr. Ziauddin Univeristy Hospital, Karachi, Pakistan
}

\author{
Correspondence: Muhammad Irfan, Faculty Office Building, National \\ Stadium Road, Aga Khan University Hospital, Karachi, Karachi City, \\ Sindh 74800, Pakistan. \\ Tel. +923002111459 . \\ E-mail: muhammad.irfan@aku.edu
}

Key words: Post TB sequelae; fibrosis; pleural thickening; bronchiectasis.

Contributions: SMZ, data collection, statistical analysis, literature review, manuscript writing; MGA, data analysis, literature review, manuscript writing; MI, study concept, manuscript reviewing and editing. All the authors have read and approved the final version of the manuscript and agreed to be accountable for all aspects of the work.

Conflict of interest: The authors declare that they have no competing interests, and all authors confirm accuracy.

Conference presentation: The abstract of this article has been presented at the following forums: - Post tuberculosis sequelae in patients treated for tuberculosis: An observational study at a tertiary care center of a high TB burden country presented at European Respiratory Society Annual Congress in September 2018 at Paris, France (Ali MG, Muhammad ZS, Shahzad T, Yaseen A, Irfan M. Post tuberculosis sequelae in patients treated for tuberculosis: An observational study at a tertiary care center of a high TB burden country). - To study the complications and sequelae in patients with pulmonary and pleural tuberculosis at a tertiary care hospital in Pakistan presented at the 49th Union World Conference on Lung Health in October 2018 at The Hague, The Netherlands. (Zubair SM, Irfan M, Gohar M. Int J Tuberc Lung Dis 2018;22(11 Suppl 2):S402.PS30-733-26). - Radiological sequelae in post tuberculosis patients treated at a tertiary care center in Pakistan: A retrospective study, presented in Virtual European Respiratory Society International Congress 2020 held in September 2020 (Zubair SM, Irfan $\mathrm{M}$, Zubairi AB. Radiological sequelae in post tuberculosis patients treated at a tertiary care center in Pakistan: A retrospective study.)

Ethical approval: This retrospective study obtained ethical approval from the Ethical Review Committee (ERC) of Aga Khan University Hospital (Application no: 5175-Med-17).

Received for publication: 1 March 2021.

Accepted for publication: 13 July 2021.

${ }^{\circ}$ Copyright: the Author(s), 2021

Licensee PAGEPress, Italy

Monaldi Archives for Chest Disease 2022; 92:1814

doi: 10.4081/monaldi.2021.1814

This article is distributed under the terms of the Creative Commons Attribution Noncommercial License (by-nc 4.0) which permits any noncommercial use, distribution, and reproduction in any medium, provided the original author(s) and source are credited.

\begin{abstract}
Treating tuberculosis (TB) is not the end of the disease because of the wide spectrum of post TB sequelae associated with the disease. There is insufficient data on post TB radiological sequelae. The aim of this study is to evaluate the post TB radiological sequelae on chest $\mathrm{x}$-rays in patients who had completed the treatment for pulmonary and pleural TB at a tertiary care hospital of a high TB burden country. This is a retrospective cross-sectional study conducted on patients treated for pulmonary and pleural TB. Adult patients (18 years or above) with a clinical or microbiological diagnosis of pulmonary or pleural TB were included. Patients were classified on the basis of site of TB into pulmonary and pleural TB. Post-treatment radiological sequelae on chest $\mathrm{x}$ ray were evaluated and divided into three main types i.e. fibrosis, bronchiectasis and pleural thickening. During the study period a total of 321 patients were included with a mean age of $44(\mathrm{SD} \pm 19)$ years. Only $17.13 \%(\mathrm{n}=55)$ patients had normal chest $\mathrm{x}$-rays at the end of treatment and $82.87 \%(\mathrm{n}=266)$ patients had post-TB radiological sequelae with fibrosis being the most common followed by pleural thickening. The post TB radiological sequelae were high in patients who had diabetes mellitus (78.94\%), AFB smear-positive (90.19\%), AFB culture-positive (89.84\%), Xpert MTB/Rif positive $(88.40 \%)$ and with drug-resistant TB $(100 \%)$. As a clinician, one should be aware of all the post TB sequelae so that early diagnosis and management can be facilitated.
\end{abstract}

\section{Introduction}

Tuberculosis (TB), caused by Mycobacterium tuberculosis (MTB), is one of the most ancient and endemic organisms worldwide. The epidemiology varies significantly with highest rates seen in Southeast Asia and sub Saharan Africa. TB is rated as one of the top ten causes of mortality worldwide and the leading cause of death in HIV patient. Pakistan is ranked as fifth amongst the high TB burden countries and also has a high prevalence of multidrug-resistant TB (MDR-TB) throughout the world [1]. TB can involve any organ of the body with variable radiological manifestations. Khan et al. elaborated the spectrum of radiological sequelae in patients already treated for pulmonary tuberculosis and has classified them into parenchymal, airway disease, pleural, mediastinal and vascular [2]. Even after treatment, patients continue to present with different symptoms and a variety of radiological sequelae. There is limited data on the radiological sequelae of tuberculosis after its cure. Previous study conducted in India shows $40.36 \%$ residual lung lesions in TB patients after it has 
been successfully treated with $59.64 \%$ showing complete resolution [3]. Radiological sequelae were also seen in $100 \%$ of the patients who were successfully treated for multidrug-resistant TB (MDR-TB) [4]. The aim of this study is to evaluate the radiological sequelae, and the extent of lung involvement at the end of treatment for pulmonary tuberculosis (PTB) and pleural tuberculosis at a tertiary care hospital in a high TB burden country, so that the whole radiological spectrum can be understood and diagnosis and management of these complications can be facilitated timely, so as to improve the quality of life of these sufferers.

\section{Materials and Methods}

This is a retrospective study conducted in patients who were diagnosed and treated as PTB and pleural TB at outpatient pulmonology clinics of Aga Khan University hospital (AKUH) from January 2014 to August 2017. The research protocol of this study was approved by the Ethical Review Committee of AKUH. We included adult patients (18 years or above) with clinical or microbiological diagnosis of pulmonary or pleural TB.

Out of 541 patients, $220(42.2 \%)$ patients were excluded. Of the excluded 220 patients, $168(76.36 \%)$ patients had no chest $\mathrm{x}$ ray available on treatment completion and 52 (23.63\%) had extra pulmonary TB other than pleural TB. Information was collected from outpatient pulmonology database and Health Information Management Services (HIMS) of the hospital. Data on demographics, comorbid conditions, site of TB, type of patient, microbiology, outcome, imaging and radiological sequelae on chest Xray was collected in a predesigned performa. Chest X-rays were evaluated by two independent pulmonologists. Patients were classified based on site of TB into PTB and pleural TB. Results of AFB smear, AFB culture and Xpert MTB/Rif were analysed. Post treatment radiological sequelae on chest $\mathrm{X}$-ray were evaluated and divided into three main types, i.e., fibrosis, bronchiectasis and pleural thickening.
Fibrosis has been explained as thickened interstitial structures and interlobular septa with reduced lung volumes of the affected regions, diaphragmatic elevation on the affected side, fibrotic bands present in the pleura and evidence of traction bronchiectasis. Bronchiectasis has been defined as bronchial walls that have thickened with single, parallel or thin lines. Sometimes tram-track sign can also be seen. Pleural thickening in a chest radiography has been defined as involvement of pleura that forms a pleural peel with relatively smoother margins, approximately $2 \mathrm{~cm}$ less in thickness. Radiologically pleural thickening can also be defined as smooth pleural opacity with involvement of at least one fourth of the chest wall circumference that obliterates the costophrenic sulcus and can also involve the apices [5,6].

All statistical analysis was done using the SPSS (Release 19.0, standard version, SPSS $^{\odot} ;$ 1989-2002). A descriptive analysis was performed for demographic features presented as mean \pm SD for quantitative variable like age. Percentage for qualitative variables like post TB sequelae, AFB smear and culture positivity, Xpert MTB/Rif positivity, associated comorbid diseases were carried out. Comparisons were made between post TB sequelae with drug resistant $\mathrm{TB}$ and presence of diabetes mellitus (DM) using chi square test.

\section{Results}

During the study period a total of 541 patients were diagnosed as PTB or pleural TB at outpatient pulmonology clinics. A total of 321 patients were included who fulfilled the inclusion criteria with a mean age of $44(\mathrm{SD} \pm 19)$ years (Figure 1). There were $60.4 \%$ $(\mathrm{n}=194)$ male and $39.5 \%(\mathrm{n}=127)$ female. Two peaks in age groups were seen one in young adults after 20 years of age and the second one in relatively old age group after 60 years.

A total of $233(72.5 \%)$ patients had pulmonary TB and 88 (27.4\%) had pleural TB. The most common associated disease was DM present in $38(11.8 \%)$ patients followed by malignancy 16

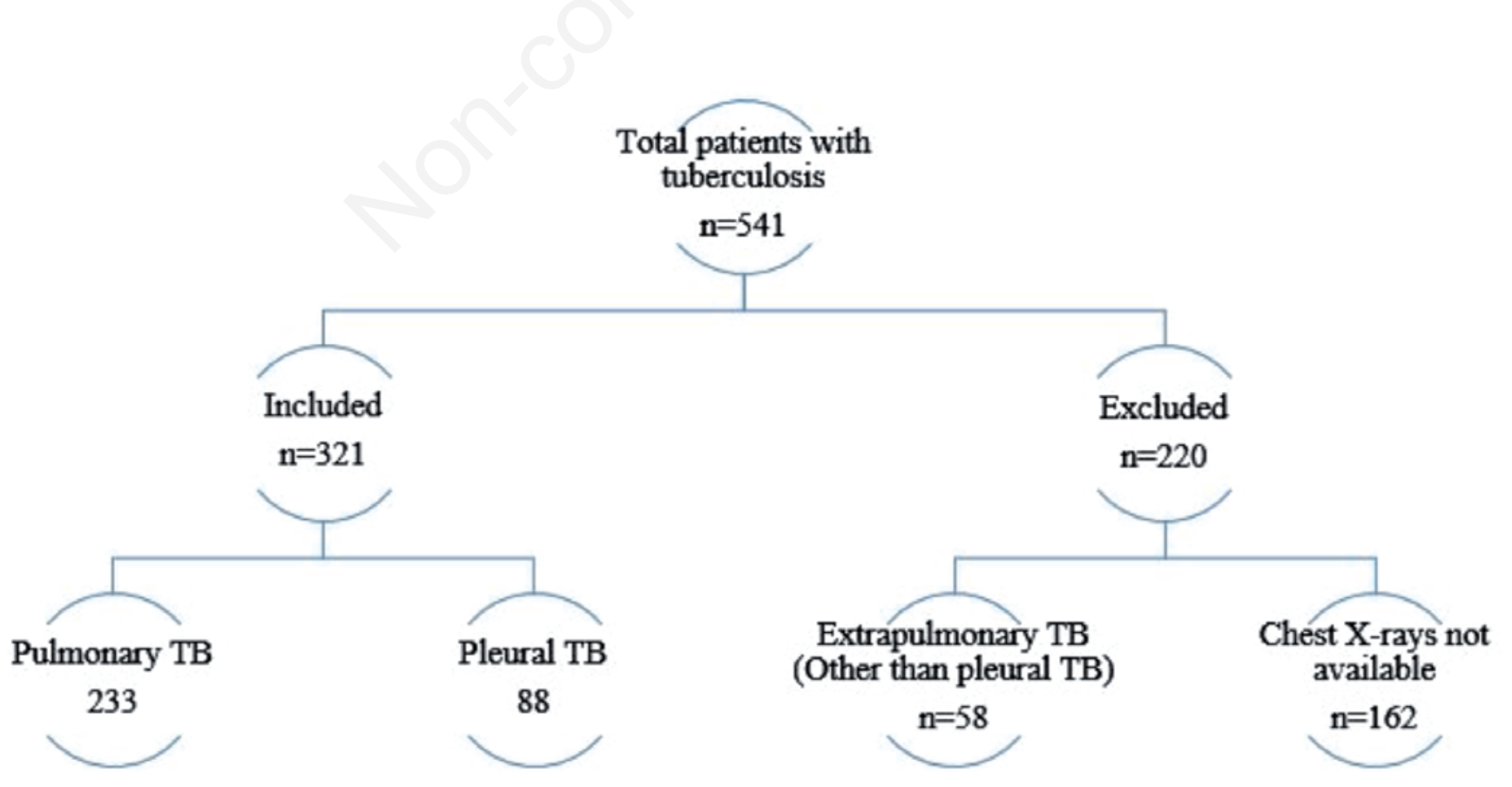

Figure 1. Patients' enrollement. 
(4.9\%) and Chronic liver disease 11 (3.4\%). Majority of the patients $(n=185,57.6 \%)$ had no prior known co-morbid conditions.

Sputum and pleural fluid AFB smears were positive in $100 / 233(42.9 \%)$ and $2 / 88(2.2 \%)$ patients respectively. Sputum AFB cultures were positive in $113 / 233(48.5 \%)$ patients. Out of these 113 positive culture patients, $97(85.8 \%)$ were pan-sensitive to all first line anti TB drugs, 12 (10.6\%) were MDR TB, $3(2.6 \%)$ were mono drug resistance, $2(1.7 \%)$ were pre-XDR and $1(0.8 \%)$ was XDR TB. Pleural fluid AFB cultures were positive in $15 / 88$ (17.04\%) patients. Out of these 15 patients, $14(93.3 \%)$ were pan-

Table 1. Demographics of the patients along with microbiological characteristics.

\begin{tabular}{lc} 
Patient characteristic & Total \\
Age (mean) years & $44 \pm 19$ \\
Male & $194(60.4 \%)$ \\
Female & $127(39.5 \%)$ \\
Associated diseases & \\
Diabetes mellitus & $38(11.8 \%)$ \\
Malignancy & $16(4.9 \%)$ \\
Chronic liver disease & $11(3.4 \%)$ \\
\hline Microbiological characteristics & \\
Sputum & \\
Sputum AFB smear positivity & $100 / 233$ \\
Sputum AFB culture positivity & $113 / 233$ \\
Pan-sensitive & $97 / 113(85.8 \%)$ \\
MDR & $12 / 113(10.6 \%)$ \\
Mono-drug resistance & $3 / 113(2.6 \%)$ \\
Pre-XDR & $2 / 113(1.7 \%)$ \\
XDR & $1 / 113(0.8 \%)$ \\
Pleural fluid & \\
Pleural fluid AFB smear positivity & $2 / 88$ \\
Pleural fluid AFB culture positivity & $15 / 88$ \\
Pan-sensitive & $14 / 15(93.3 \%)$ \\
Mono-drug resistance & $1 / 15(6.6 \%)$ \\
\hline Nucleic acid amplification test & \\
Xpert MTB /Rif positivity in sputum & $127 / 233$ \\
Xpert MTB /Rif positivity in pleural fluid & $11 / 88$ \\
\hline
\end{tabular}

Table 2. Frequency and types of post-TB sequelae in our population.

\begin{tabular}{lc}
\hline Radiological sequelae & $\mathrm{n}=321(\%)$ \\
Normal & $55(17.13)$ \\
Parenchymal sequelae & \\
Unilateral bronchiectasis & $9(2.80)$ \\
Bilateral bronchiectasis & $1(0.31)$ \\
Unilateral bronchiectasis and fibrosis & $10(3.11)$ \\
Bilateral bronchiectasis and fibrosis & $3(0.93)$ \\
Unilateral fibrosis & $112(34.89)$ \\
Bilateral fibrosis & $37(11.52)$ \\
\hline Pleural sequelae & \\
Unilateral pleural thickening & $65(20.24)$ \\
Bilateral pleural thickening & $17(5.29)$ \\
Pleuro-parenchymal sequelae & \\
Unilateral pleural thickening and bronchiectasis & $2(0.62)$ \\
Unilateral pleural thickening with bronchiectasis and fibrosis $4(1.24)$ \\
Bilateral pleural thickening and bronchiectasis \\
Bilateral pleural thickening with fibrosis and bronchiectasis & $5(1.55)$ \\
\hline
\end{tabular}

sensitive and $1(6.6 \%)$ showed mono-drug resistance. Sputum and pleural fluid Xpert MTB /Rif was positive in 127/233 (54.5\%) and $11 / 88$ (12.5\%) patients respectively. Demographic and microbiological data has been described in Table 1. Out of 321 patients, 298 $(92.8 \%)$ were new cases and $23(7.16 \%)$ were relapse. At the end of treatment $303(94.3 \%)$ were labelled as cured and $18(5.6 \%)$ had treatment failure. Out of 321 patients, only $17.13 \%(n=55)$ patients had normal chest X-rays at the end of treatment and $82.87 \%$ $(n=266)$ patients had post TB radiological sequelae. The radiological pattern of the post treatment sequelae is shown in Table 2.

We also analyzed the relation of post treatment radiological sequelae in patients who had AFB smear positive, Xpert MTB/Rif positive and AFB culture positive. Sputum and pleural fluid AFB smear were positive in 102/321 (31.7\%) patients. Out of these 102 patients, only $10(9.8 \%)$ had normal chest x-ray and $92(90.1 \%)$ had radiological sequelae after completion of treatment. Fibrosis was the most common sequelae. Sputum and pleural fluid Xpert MTB/RIF was positive in 138/321 (42.9\%) patients. Out of these 138 patients, only $16(11.5 \%)$ had normal chest x-ray and 122 $(88.4 \%)$ had radiological sequelae after completion of treatment. Sputum and pleural fluid AFB cultures were positive in $128 / 321$ (39.8\%) patients. Out of these 128 patients, only $13(10.1 \%)$ had normal chest X-ray and $115(89.8 \%)$ had radiological sequelae after completion of treatment. The relation of development of post TB sequelae with smear, culture and Xpert MTB/RIF positivity are presented in Figure 2. Out of 128 AFB culture (sputum and pleural fluid) positive cases, 19 patients had drug resistance (MDR, pre XDR, XDR, Mono-drug resistance and polydrug resistance). All of these $19(100 \%)$ patients showed post TB radiological sequelae. The relation of Post TB radiological sequelae with the concomitant presence of diabetes was also analysed. We had total 38 patients who had diabetes mellitus as a comorbid condition. Out of these 38 patients, $n=8(21.05 \%)$ had complete resolution on their chest $\mathrm{x}$ rays however $n=30(78.94 \%)$ patients had some kind of post TB sequelae which are described in Figure 3.

\section{Discussion}

Post TB sequelae has always been underestimated [7]. We validated that in our population, post TB sequelae is very high $(82.87 \%)$ with unilateral fibrosis $(34.89 \%)$ being the most com-

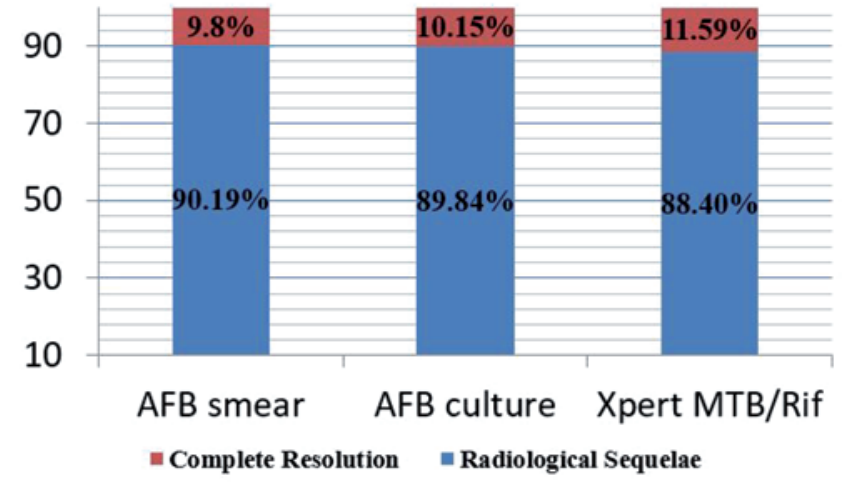

Figure 2. Frequency of post-TB sequelae in patients who are AFB smear, AFB culture and Xpert/MTB Rif positive in sputum and pleural fluid samples. 
mon one. We also validated that the frequency of post TB sequelae was also very high in patients who were AFB smear positive, AFB culture positive and Xpert/MTB Rif positive. Our analysis emphasizes upon the fact that there are greater chances of post TB sequelae in patients who are AFB smear and culture positive hence the job remains incomplete even after the treatment, because majority of the patients will present with debilitating symptoms.

In our study, we found that post TB sequelae of one type or another are more significant in drug resistant TB and none of the patients showed complete resolution on chest radiography. Furthermore, the patients with concomitant TB and DM also had high incidence of post TB sequelae.

Apart from symptoms, tuberculosis after being successfully treated has great impact on the quality of life of patients because of the stigma it carries with it. Rajeswari et al. studied the effects of tuberculosis on the social, physical and mental wellbeing of patients and described that social stigma persisted in both men and women even after they were completely treated [8]. Despite adequate treatment, TB can cause significant health issues which are short term and long term both. Complications of TB disease are attributed to structural and vascular compromise caused by Mycobacterium tuberculosis [9]. Multiple forms of sequelae and complications may result from pulmonary TB in both treated and untreated patients. In fact, complication can be the initial presentation of Pulmonary TB in

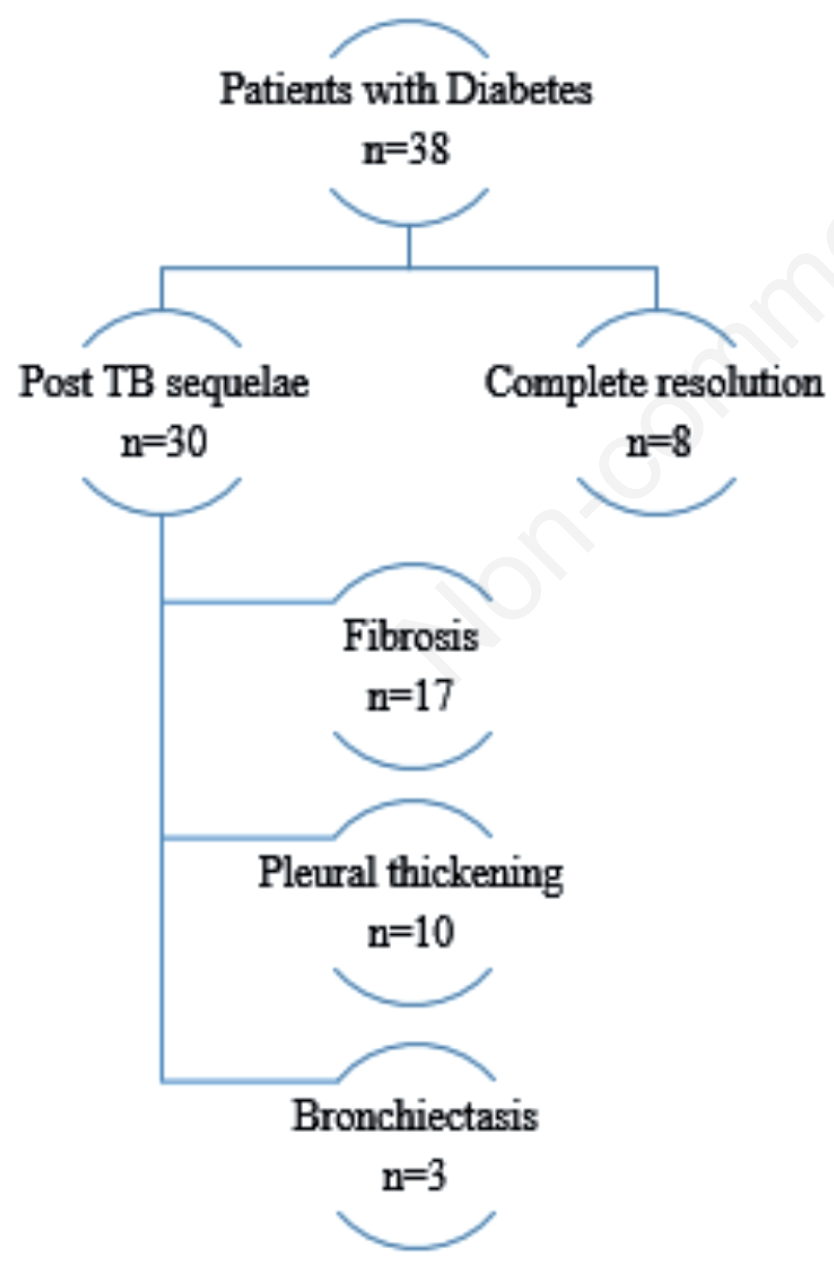

Figure 3. Frequency and types of post-TB sequelae in patients with diabetes mellitus. certain cases [10]. Satish et al. analysed CT scan chest of 100 patients for post-TB sequelae. They categorized the sequelae into parenchymal, airway, pleural, mediastinal, and vascular subtypes. Fibrosis was seen as the most common sequelae in their cohort [11]. Menon et al. have described the radiological sequelae in new cases of pulmonary, pleural and mediastinal tuberculosis after they completed their treatment. In their study complete resolution of lesions were observed in $59.6 \%$ of patients with $38.7 \%$ showing fibrosis [3]. However, in our population only $17.1 \%$ patients had completely normal chest-x-ray after treatment completion with $46.41 \%$ showing some fibrosis. A systemic review done in United Kingdom demonstrated the differences in the patterns and prevalence of lung damage seen using chest X-ray and computed tomography scan in post-TB patients. Chest X-ray focused on cavitation, bronchiectasis, and fibrosis, out of which cavitation was reported as the most common finding. In CT scan bronchiectasis was more common than cavitation with features of inflammation, emphysema, and mosaicism. The facility of computed tomographic imaging's availability remains restricted and limited in low to middle income countries hence the post TB sequelae are underestimated and under reported [7].

Singla et al. studied the radiological sequelae in patients treated for MDR TB. After conclusion of two years of MDR-TB treatment $95.7 \%$ patients had some residual symptoms and $100 \%$ patients had residual abnormality on bilateral chest $\mathrm{x}$-ray with $82.6 \%$ patients showing disease in advanced stage [4]. Our study also analysed the residual chest $\mathrm{x}$-ray findings in patients treated for drug resistant tuberculosis and it was seen that $100 \%$ patients had post tuberculosis radiological sequelae of one kind or another. Singla et al. [12] also studied residual radiological sequelae in total 51 MDR-TB patients who were treated successfully. Out of these $98 \%$ had residual radiological sequelae while $78 \%$ had persistent respiratory symptoms.

Bronchiectasis has always been regarded as "orphan lung disease" [13]. It is known to be a common post TB sequela. A study was done in Nepal to see the clinical spectrum of patients who presented with bronchiectasis. Post-TB bronchiectasis was found to be the most common cause [14]. Lower lobes tend to have more retained secretions with increased bronchial pressures and infections making it the most suitable site for the development of bronchiectasis. In comparison, the upper lobes tend to have very few secretions [15]. In our study bronchiectasis was seen in $10.87 \%$ of patients. There is a possibility of underestimation because data was analysed on the basis of chest X-rays and highly resonant computed tomography scan of the chest were not available to properly evaluate the bronchiectatic changes.

TB has always been associated with certain atypical manifestations in diabetic patients. Shaikh et al found out that patients with concomitant pulmonary TB and diabetes mellitus are more likely to present with atypical radiological images including cavitatory lesions and predominance of the lower lung [16]. Patel et al. also found that there was a greater involvement of lower lungs (84\%) as compared to upper lungs $(16 \%)$. Moreover, they also found out that unilateral involvement was $68 \%$ while bilateral involvement was $32 \%$. Out of 50 patients, 10 patients had cavitatory disease which were frequent in the lower lung zone $(80 \%)$ [17]. Although the relation between radiological manifestation of pulmonary TB and diabetes mellitus has been established in literature, there is limited data describing the effect of post-TB sequelae in diabetic patients. We analysed 38 patients with concomitant diabetes and TB. We found out that 30 (78.94\%) patients developed radiological sequelae. Unilateral involvement was seen in $79.41 \%$ and bilateral involvement was seen in $20.58 \%$ of the patients. Fibrosis $(56.6 \%)$ was seen as the most common radiolog- 
ical sequelae followed by pleural thickening $10(33.33 \%)$ and bronchiectasis $3(10 \%)$.

Post-TB sequelae has certain clinical implications. The frequently observed symptoms in such patients are dyspnoea, cough, wheezing and sputum production [18]. Chronic bronchitis, exacerbations of chronic obstructive pulmonary disease and bronchiectasis are also a known complications affecting the post-TB lungs. Among the infective complications, Nontuberculous mycobacteria and Aspergillus causing chronic pulmonary aspergillosis are now considered the most common pathogens affecting the diseased lungs $[7,19,20]$. Di Naso et al. described that patients who have received multiple treatments for TB are more prone to develop post-TB sequelae associated with significantly lower forced vital capacity, MIP and distance covered in 6-minute walk test [21]. A cross sectional study in South Africa showed that patient cured for TB show decreased exercise capacity and health related quality of life along with abnormal lung functions [22]. Chronic pulmonary function impairment was assessed in another South African population and it was seen that lung functional impairment increases incrementally with the number of episodes of TB [23]. Although post-TB sequelae once diagnosed on chest $\mathrm{x}$-rays and CT scans may remain asymptomatic but some patients presenting with symptoms (breathlessness, cough, chronic respiratory failure, haemoptysis) needs follow up assessment with sputum cultures, fungal markers, lung function tests and 6-minute walk test so that disease progression can be diagnosed and management can be facilitated timely. Post-TB patients with lung functional abnormalities, reduced exercise capacity and impaired quality of life should ideally undergo pulmonary rehabilitation which can be beneficial as discussed by Visca et al. [24,25].

Our study had few limitations. The study had high number of cases which were excluded because of multiple factors like unavailability of chest X-rays and diagnosis of TB other than pulmonary and pleural TB. We evaluated the chest X-rays for determining the frequency of Post TB radiological sequelae, however for sequelae like bronchiectasis computed tomography scan of the chest is the best modality which was not available for our patients. Majority of patients presented with certain symptoms like cough, breathlessness on mild to moderate exertion, wheezing but we did not specifically categorize the symptoms with which they presented. Certain studies suggest the deteriorating pulmonary function tests after tuberculosis has been treated. We did not analyse the pulmonary functions and how they deteriorate in post-TB sequelae.

\section{Conclusions}

The post TB radiological sequelae in our cohort is very high. Hence treating tuberculosis is not the end of this epidemic. The comorbidities associated with post tuberculosis sequelae continues to cripple the life of the sufferers. It has effects not only on the health of the patient but also physical, social, mental and economic aspects of life. Hence as a physician one should be aware of all the spectrum of the sequelae so that early diagnosis and management can be facilitated.

\section{References}

1. World Health Organization. Global tuberculosis report 2018: World Health Organization; 2018.
2. Khan R, Malik NI, Razaque AJP. Imaging of pulmonary posttuberculosis sequelae. Pak J Med Sci 2020;36:S75.

3. Menon B, Nima G, Dogra V, Jha S. Evaluation of the radiological sequelae after treatment completion in new cases of pulmonary, pleural, and mediastinal tuberculosis. Lung India 2015;32:241-5.

4. Singla R, Mallick M, Mrigpuri P, et al. Sequelae of pulmonary multidrug-resistant tuberculosis at the completion of treatment. Lung India 2018;35:4-8.

5. Proschek P, Vogl TJ. Chest and mediastinum. In: T Vogl, W Reith, E Rummeny, Editors. Diagnostic and Interventional Radiology. Berlin: Springer; 2015. p. 479-587.

6. Stark P. Imaging in Pulmonary Disease. In: L Goldman, A Schafer, Editors. Goldman's Cecil medicine. Amsterdam: Elsevier; 2012. Chapter 84.

7. Meghji J, Simpson H, Squire SB, Mortimer K. A systematic review of the prevalence and pattern of imaging defined postTB lung disease. PLoS One 2016;11:e0161176-e.

8. Rajeswari R, Muniyandi M, Balasubramanian R, Narayanan PR. Perceptions of tuberculosis patients about their physical, mental and social well-being: a field report from south India. Soc Sci Med 2005;60:1845-53.

9. Shah M, Reed C. Complications of tuberculosis. Curr Opin Infect Dis 2014;27:403-10.

10. Satya SS. Textbook of pulmonary and extra pulmonary tuberculosis. Mehta Publishers; 2009.

11. Deshpande SS, Joshi AR, Shah AJP. Aftermath of pulmonary tuberculosis: computed tomography assessment. Pol J Radiol 2020;85:e144-54.

12. Singla N, Singla R, Fernandes S, Behera D. Post treatment sequelae of multi-drug resistant tuberculosis patients. Indian $\mathrm{J}$ Tubercul 2009;56:206-12.

13. Barker AF. Bronchiectasis. N Engl J Med. 2002;346:1383-93.

14. Bhatta N, Dhakal S, Rizal S, et al. Clinical spectrum of patients presenting with bronchiectasis in Nepal: evidence of linkage between tuberculosis, tobacco smoking and toxic exposure to biomass smoke. Kathmandu University Medical Journal (KUMJ) 2008;6:195-203.

15. Salkin D. Tuberculosis as a cause of upper lobe bronchiectasis. Califor Med 1950;73:577.

16. Shaikh MA, Singla R, Khan NB, et al. Does diabetes alter the radiological presentation of pulmonary tuberculosis. Saudi Med J 2003;24:278-81.

17. Patel AK, Rami KC, Ghanchi FD. Radiological presentation of patients of pulmonary tuberculosis with diabetes mellitus. Lung India 2011;28:70.

18. Nihues SdSE, Mancuzo EV, Sulmonetti N, et al. Chronic symptoms and pulmonary dysfunction in post-tuberculosis Brazilian patients. Braz J Infect Dis 2015;19:492-7.

19. Hsu D, Irfan M, Jabeen K, et al. Post tuberculosis treatment infectious complications. Int J Infect Dis 2020;92:S41-5.

20. Denning DW, Pleuvry A, Cole DC. Global burden of chronic pulmonary aspergillosis as a sequel to pulmonary tuberculosis. Bull World Health Organ 2011;89:864-72.

21. Di Naso FC, Pereira J, Schuh S, Unis G. [Functional evaluation in patients with pulmonary tuberculosis sequelae].[Article in Portuguese]. Rev Port Pneumol 2011;17:216-21.

22. Hanekom S, Pharaoh H, Irusen E, Daniels KJ. Post-tuberculosis health-related quality of life, lung function and exercise capacity in a cured pulmonary tuberculosis population in the Breede Valley District, South Africa. S Afr J Physiother 2019;75:1319.

23. Hnizdo E, Singh T, Churchyard G. Chronic pulmonary func- 
tion impairment caused by initial and recurrent pulmonary tuberculosis following treatment. Thorax 2000;55:32-8.

24. Visca D, Centis R, D'Ambrosio L, et al. The need for pulmonary rehabilitation following tuberculosis treatment. Int $\mathrm{J}$
Tuberc Lung Dis 2020;24:720-2.

25. Visca D, Zampogna E, Sotgiu G, et al. Pulmonary rehabilitation is effective in patients with tuberculosis pulmonary sequelae. Eur Respir J 2019;53:1802184. 